\title{
On a local version of Jack's lemma
}

\author{
Mamoru Nunokawa, Janusz Sokół
}

\begin{abstract}
The purpose of this paper is to provide a result which concerns with the boundary behavior of analytic functions. It may be a local version of the well known Jack's lemma when we change the function normalization at the origin.
\end{abstract}

\section{Introduction}

Let $\mathcal{H}$ denote the class of analytic functions in the unit disk $\mathbb{D}=\{z \in \mathbb{C}$ : $|z|<1\}$. Let $\mathcal{A}(p)$ denote the class of all functions analytic in the unit disk $\mathbb{D}$ which have the form

$$
f(z)=z^{p}+\sum_{n=1}^{\infty} a_{p+n} z^{p+n}, \quad z \in \mathbb{D},
$$

where $p$ is positive integer. In this section we develop a key lemma that forms the groundwork for many of the results. It is a local version of the following lemma, well known as the Jack's lemma.

Lemma 1.1. [1] Let $w(z)$ be non-constant and analytic function in the unit disc $\mathbb{D}$ with $w(0)=0$. If $|w(z)|$ attains its maximum value on the disc $|z| \leq r$ at the point $z_{0},\left|z_{0}\right|=r$, then $z_{0} w^{\prime}\left(z_{0}\right)=k w\left(z_{0}\right)$ and $k \geq 1$.

The Jack's lemma has found several of the applications and generalizations in the theory of differential subordinations, see for instance [2], [3] and [4]. In this paper we generalize the following Nunokawa's lemma, [5], see also [6] for its angle version.

Key Words: convex, starlike, analytic functions, univalent functions, Jack's lemma.

2010 Mathematics Subject Classification: Primary 30C45, Secondary 30C80.

Received: 01.03.2018

Accepted: 24.09.2018. 
Lemma 1.2. Let $p$ be analytic function in $|z|<1$, with $p(0)=1$. If there exists a point $z_{0},\left|z_{0}\right|<1$, such that $\mathfrak{R e}\{p(z)\}>0$ for $|z|<\left|z_{0}\right|$ and $p\left(z_{0}\right)= \pm$ ia for some $a>0$, then we have

$$
\frac{z_{0} p^{\prime}\left(z_{0}\right)}{p\left(z_{0}\right)}=\frac{2 i k \arg \left\{p\left(z_{0}\right)\right\}}{\pi}, \quad \arg \left\{p\left(z_{0}\right)\right\}= \pm \frac{\pi}{2}
$$

for some $k \geq\left(a+a^{-1}\right) / 2 \geq 1$.

LemmA 1.3. Let $p(z)=1+\sum_{n=1}^{\infty} c_{n} z^{n}$ be analytic in $\mathbb{D}$ with $p(z) \neq 0$ therein. If there exists a point $z_{1}, 0<\left|z_{1}\right|<1$ and the sector $S_{\delta}\left(z_{1}\right)$, for which

$$
\max \left\{z \in S_{\delta}\left(z_{1}\right):|p(z)|\right\}=\left|p\left(z_{1}\right)\right|
$$

where $z_{1}=\left|z_{1}\right| e^{i \theta_{1}}$

$$
S_{\delta}\left(z_{1}\right)=\left\{r e^{i \theta}: 0 \leq r \leq\left|z_{1}\right|,\left|\theta-\theta_{1}\right| \leq \delta\right\},
$$

then we have

$$
\frac{z_{1} p^{\prime}\left(z_{1}\right)}{p\left(z_{1}\right)} \in \mathbb{R}, \quad \frac{z_{1} p^{\prime}\left(z_{1}\right)}{p\left(z_{1}\right)} \geq 0,
$$

moreover

$$
\mathfrak{R e}\left\{1+\frac{z_{1} p^{\prime \prime}\left(z_{1}\right)}{p^{\prime}\left(z_{1}\right)}\right\} \geq \frac{z_{1} p^{\prime}\left(z_{1}\right)}{p\left(z_{1}\right)} \geq 0 .
$$
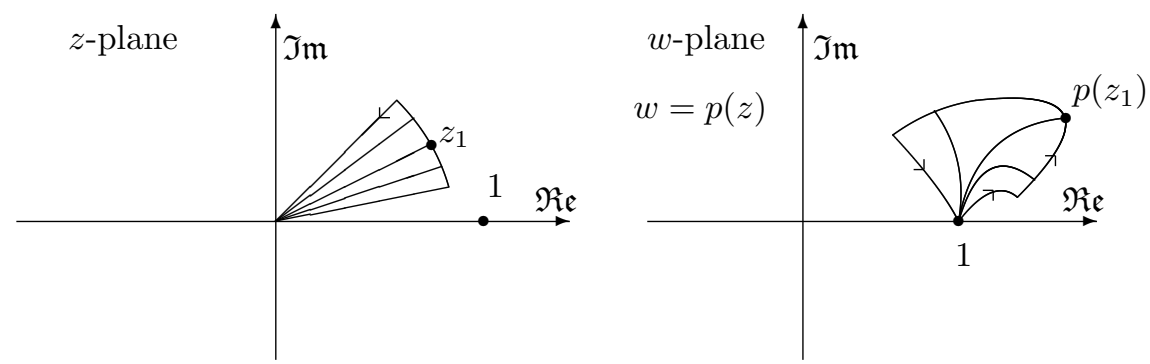

Fig.1. $z$-plane.

Fig.2. $w$-plane.

Proof. From the hypothesis, we can have the above pictures, Fig. 1. and Fig. 2. Then it follows that

$$
\frac{z p^{\prime}(z)}{p(z)}=\frac{\mathrm{d} \log |p(z)|+i \mathrm{~d} \arg \{p(z)\}}{i \mathrm{~d} \theta}=\frac{\mathrm{d} \arg \{p(z)\}}{\mathrm{d} \theta}-i \frac{1}{|p(z)|} \frac{\mathrm{d}|p(z)|}{\mathrm{d} \theta},
$$


where $z$ moves on the arc $z=\left|z_{1}\right| e^{i \theta}$ and $\theta_{1}-\delta \leq \theta \leq \theta_{1}+\delta$. From the hypothesis, we have also

$$
\left(\frac{\mathrm{d}|p(z)|}{\mathrm{d} \theta}\right)_{z=z_{1}}=0
$$

and from geometrical observation, we have

$$
\left(\frac{\mathrm{d} \arg \{p(z)\}}{\mathrm{d} \theta}\right)_{z=z_{1}} \geq 0 .
$$

It completes the proof of (3). To prove (4) let us put

$$
q(z)=\frac{z p^{\prime}(z)}{p(z)}, \quad q(0)=0 .
$$

From the hypothesis, $q(z)$ is analytic in $\mathbb{D}$ and

$$
q(z) \neq 0, \quad z \in S_{\delta}\left(z_{1}\right)
$$

Then it follows that

$$
q(z)=\frac{z p^{\prime}(z)}{p(z)}=\frac{\mathrm{d} \arg \{p(z)\}}{\mathrm{d} \theta}-i \frac{1}{|p(z)|} \frac{\mathrm{d}|p(z)|}{\mathrm{d} \theta}
$$

where $z=\left|z_{1}\right| e^{i \theta}$ and $\theta_{1}-\delta \leq \theta \leq \theta_{1}+\delta$. Then, from the above picture, we have

$$
\frac{\mathrm{d}|p(z)|}{\mathrm{d} \theta} \geq 0, \quad \theta_{1}-\delta \leq \theta \leq \theta_{1}
$$

and

$$
\frac{\mathrm{d}|p(z)|}{\mathrm{d} \theta} \leq 0, \quad \theta_{1} \leq \theta \leq \theta_{1}+\delta
$$

Therefore, we have

$$
\begin{aligned}
& \operatorname{Im}\{q(z)\})<0 \text { for } \theta_{1}-\delta \leq \theta \leq \theta_{1}, \\
& \mathfrak{I m}\{q(z)\})=0 \text { for } \theta=\theta_{1}, \\
& \operatorname{Im}\{q(z)\})>0 \text { for } \theta_{1} \leq \theta \leq \theta_{1}+\delta .
\end{aligned}
$$

This shows that

$$
\begin{aligned}
\left(\frac{\mathrm{d} \arg \{q(z)\}}{\mathrm{d} \theta}\right)_{z=z_{1}} & =\mathfrak{R e}\left\{\frac{z q^{\prime}(z)}{q(z)}\right\}_{z=z_{1}} \\
& =\mathfrak{R e}\left\{1+\frac{z p^{\prime \prime}(z)}{p^{\prime}(z)}-\frac{z p^{\prime}(z)}{p(z)}\right\}_{z=z_{1}} \\
& \geq 0
\end{aligned}
$$


This shows that

$$
1+\mathfrak{R e}\left\{1+\frac{z_{1} p^{\prime \prime}\left(z_{1}\right)}{p^{\prime}\left(z_{1}\right)}\right\} \geq \mathfrak{R e}\left\{\frac{z_{1} p^{\prime}\left(z_{1}\right)}{p\left(z_{1}\right)}\right\}=\frac{z_{1} p^{\prime}\left(z_{1}\right)}{p\left(z_{1}\right)} .
$$

It completes the proof of (4).

Remark The results of Lemma 1.3 and Theorem 2.1 below, hold to be correct not only for the case $|p(z)|$ and $|f(z)|$ take its local maximum value at the point $z=z_{0}$ in the domain $|z| \leq\left|z_{0}\right|$ but at the point $z_{1}$ in the subset $S_{\delta}\left(z_{1}\right) \subset \mathbb{D}$. It is an improvement of the known results from [1] and [4]. Lemma 1.3 is applicable for the points $z=\alpha$ and not for $z=\beta$, Fig. 3 .

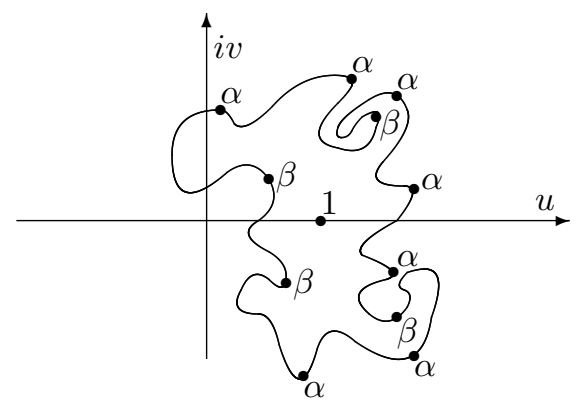

Fig.3. $p\left(|z| \leq\left|z_{1}\right|\right)$.

\section{Applications}

TheOREM 2.1. Let $f(z)=z^{p}+\sum_{n=p+1}^{\infty} a_{n} z^{n}, 1 \leq p$, be analytic and p-valent in $\mathbb{D}$. If there exists a point $z_{1}, 0<\left|z_{1}\right|<1$ and the sector $S_{\delta}\left(z_{1}\right)$, for which

$$
\max \left\{z \in S_{\delta}\left(z_{1}\right):|f(z)|\right\}=\left|f\left(z_{1}\right)\right|,
$$

where $z_{1}=\left|z_{1}\right| e^{i \theta_{1}}$ and

$$
S_{\delta}\left(z_{1}\right)=\left\{r e^{i \theta}: 0 \leq r \leq\left|z_{1}\right|,\left|\theta-\theta_{1}\right|<\delta\right\},
$$

then we have

$$
\frac{z_{1} f^{\prime}\left(z_{1}\right)}{f\left(z_{1}\right)} \in \mathbb{R}, \quad \frac{z_{1} f^{\prime}\left(z_{1}\right)}{f\left(z_{1}\right)} \geq p
$$


moreover

$$
\mathfrak{R e}\left\{1+\frac{z_{1} f^{\prime \prime}\left(z_{1}\right)}{f^{\prime}\left(z_{1}\right)}\right\} \geq \mathfrak{R e}\left\{\frac{z_{1} f^{\prime}\left(z_{1}\right)}{f^{\prime}\left(z_{1}\right)}\right\}=\frac{z_{1} f^{\prime}\left(z_{1}\right)}{f^{\prime}\left(z_{1}\right)} \geq p .
$$

Proof. For the proof of (10), let us put

$$
p(z)=\frac{f(z)}{z^{p}}, \quad p(0)=1 .
$$

From the hypothesis, we have that $p(z)$ is analytic in $\mathbb{D}$ and $p(z) \neq 0$ in $\mathbb{D}$ since $f(z)$ is $p$-valent in $\mathbb{D}$. Then it follows that $|p(z)|$ takes its maximum value at the point $z=z_{1}$ in the sector $S_{\delta}\left(z_{1}\right)$. Therefore, applying Lemma 1.3, we have

$$
\begin{aligned}
\frac{z_{1} p^{\prime}\left(z_{1}\right)}{p\left(z_{1}\right)} & =\mathfrak{R e}\left\{\frac{z_{1} p^{\prime}\left(z_{1}\right)}{p\left(z_{1}\right)}\right\} \\
& =\frac{z_{1} f^{\prime}\left(z_{1}\right)}{f\left(z_{1}\right)}-p \\
& =\operatorname{Re}\left\{\frac{z_{1} f^{\prime}\left(z_{1}\right)}{f\left(z_{1}\right)}\right\}-p \\
& \geq 0 .
\end{aligned}
$$

It completes the proof of (10).

For the proof of (11), let us put

$$
q(z)=\frac{z f^{\prime}(z)}{p f(z)}, \quad q(0)=1 .
$$

From the hypothesis, and from $(10), q(z)$ is analytic in $\mathbb{D}$ and

$$
\frac{z_{1} f^{\prime}\left(z_{1}\right)}{f\left(z_{1}\right)} \geq p^{2}>0
$$

Applying Lemma 1.3, we have

$$
\begin{aligned}
\frac{z_{1} q^{\prime}\left(z_{1}\right)}{q\left(z_{1}\right)} & =\mathfrak{R e}\left\{1+\frac{z_{1} f^{\prime \prime}\left(z_{1}\right)}{f^{\prime}\left(z_{1}\right)}-\frac{z_{1} f^{\prime}\left(z_{1}\right)}{f\left(z_{1}\right)}\right\} \\
& =\mathfrak{R e}\left\{1+\frac{z_{1} f^{\prime \prime}\left(z_{1}\right)}{f^{\prime}\left(z_{1}\right)}\right\}-\mathfrak{R e}\left\{\frac{z_{1} f^{\prime}\left(z_{1}\right)}{f\left(z_{1}\right)}\right\} \\
& \geq 0 .
\end{aligned}
$$

this shows that

$$
1+\mathfrak{R e}\left\{\frac{z_{1} f^{\prime \prime}\left(z_{1}\right)}{f^{\prime}\left(z_{1}\right)}\right\} \geq \mathfrak{R e}\left\{\frac{z_{1} f^{\prime}\left(z_{1}\right)}{f\left(z_{1}\right)}\right\}=\frac{z_{1} f^{\prime}\left(z_{1}\right)}{f\left(z_{1}\right)} \geq 0 .
$$


It completes the proof of (11).

LEMmA 2.2. Let $p(z)=1+\sum_{n=1}^{\infty} c_{n} z^{n}$ be analytic in $\mathbb{D}$ with $p(z) \neq 0$ with $p(z) \neq 0$ therein. If there exists a point $z_{1}, 0<\left|z_{1}\right|<1$ and the sector $S_{\delta}\left(z_{1}\right)$, for which

$$
\min \{|z| \leq r<1:|p(z)|\}=\left|p\left(z_{1}\right)\right|
$$

where $\left|z_{1}\right|=r<1$. Then we have

$$
\frac{z_{1} p^{\prime}\left(z_{1}\right)}{p\left(z_{1}\right)} \in \mathbb{R}, \quad \frac{z_{1} p^{\prime}\left(z_{1}\right)}{p\left(z_{1}\right)} \leq 0,
$$

moreover

$$
\mathfrak{R e}\left\{1+\frac{z_{1} p^{\prime \prime}\left(z_{1}\right)}{p^{\prime}\left(z_{1}\right)}\right\} \leq \frac{z_{1} p^{\prime}\left(z_{1}\right)}{p\left(z_{1}\right)} \leq 0 .
$$

Proof. then we have

$$
\begin{aligned}
\frac{z_{1} p^{\prime}\left(z_{1}\right)}{p\left(z_{1}\right)} & =\left.\frac{\mathrm{d} \log p(z)}{\mathrm{d} \log z}\right|_{z=z_{1}} \\
& =\left.\frac{\mathrm{d} \log |p(z)|+i \mathrm{~d} \arg \{p(z)\}}{i \mathrm{~d} \varphi}\right|_{z=z_{1}} \\
& =\frac{\mathrm{d} \arg \{p(z)\}}{\mathrm{d} \varphi}-\left.\frac{i}{|p(z)|} \frac{\mathrm{d}|p(z)|}{\mathrm{d} \varphi}\right|_{z=z_{1}} \\
& =\left.\frac{\mathrm{d} \arg \{p(z)\}}{\mathrm{d} \varphi}\right|_{z=z_{1}} \\
& \leq 0,
\end{aligned}
$$

because of (12). This gives (13). For the proof of (14) consider

$$
\begin{aligned}
\frac{\mathrm{d} \log \left(\frac{z p^{\prime}(z)}{p(z)}\right)}{\mathrm{d} \log \{z\}} & =\frac{\mathrm{d} \log \left|\frac{z p^{\prime}(z)}{p(z)}\right|}{i \mathrm{~d} \theta}-\frac{i}{i \mathrm{~d} \theta}\left(\frac{1}{|p(z)|} \frac{\mathrm{d}|p(z)|}{\mathrm{d} \theta}\right) \\
& =-\frac{\mathrm{d}}{\mathrm{d} \theta}\left(\frac{1}{|p(z)|} \frac{\mathrm{d}|p(z)|}{\mathrm{d} \theta}\right)-i \frac{\mathrm{d}}{\mathrm{d} \theta}\left(\frac{\mathrm{d} \arg \{p(z)\}}{\mathrm{d} \theta}\right) \\
& =\frac{1}{|p(z)|^{2}}\left(\frac{\mathrm{d}|p(z)|}{\mathrm{d} \theta}\right)^{2}-\frac{1}{|p(z)|}\left(\frac{\mathrm{d}^{2}|p(z)|}{\mathrm{d} \theta^{2}}\right)-i \frac{\mathrm{d}^{2} \arg \{p(z)\}}{\mathrm{d} \theta^{2}} \\
& =1+\frac{z p^{\prime \prime}(z)}{p^{\prime}(z)}-\frac{z p^{\prime}(z)}{p(z)},
\end{aligned}
$$


where $z=r e^{i \theta}$ and $0 \leq \theta \leq 2 \pi$. If we put $z=z_{1}$, then we have

$$
\begin{aligned}
& 1+\frac{z_{1} p^{\prime \prime}\left(z_{1}\right)}{p^{\prime}\left(z_{1}\right)}-\frac{z_{1} p^{\prime}\left(z_{1}\right)}{p\left(z_{1}\right)} \\
= & \frac{1}{|p(z)|^{2}}\left(\frac{\mathrm{d}|p(z)|}{\mathrm{d} \theta}\right)_{z=z_{1}}^{2}-\frac{1}{|p(z)|}\left(\frac{\mathrm{d}^{2}|p(z)|}{\mathrm{d} \theta^{2}}\right)_{z=z_{1}}-i\left(\frac{\mathrm{d}^{2} \arg \{p(z)\}}{\mathrm{d} \theta^{2}}\right)_{z=z_{1}} \\
= & -\frac{1}{|p(z)|}\left(\frac{\mathrm{d}^{2}|p(z)|}{\mathrm{d} \theta^{2}}\right)_{z=z_{1}}-i\left(\frac{\mathrm{d}^{2} \arg \{p(z)\}}{\mathrm{d} \theta^{2}}\right)_{z=z_{1}}
\end{aligned}
$$

because of (12). Therefore,

$$
\begin{aligned}
& \mathfrak{R e}\left\{1+\frac{z_{1} p^{\prime \prime}\left(z_{1}\right)}{p^{\prime}\left(z_{1}\right)}-\frac{z_{1} p^{\prime}\left(z_{1}\right)}{p\left(z_{1}\right)}\right\} \\
= & -\frac{1}{|p(z)|}\left(\frac{\mathrm{d}^{2}|p(z)|}{\mathrm{d} \theta^{2}}\right)_{z=z_{1}} \\
\leq & 0
\end{aligned}
$$

because $|p(z)|$ attains its minimum value at $z=z_{1}$, and from the known geometric property, we have

$$
\left(\frac{\mathrm{d}^{2}|p(z)|}{\mathrm{d} \theta^{2}}\right)_{z=z_{1}} \geq 0
$$

It completes the proof of (14).

Applying Lemma 2.2 and the same method as in the proof of Theorem 2.1 we can proof the following theorem.

TheOREM 2.3. Let $f(z)=z^{p}+\sum_{n=p+1}^{\infty} a_{n} z^{n}, 1 \leq p$, be analytic and p-valent in $\mathbb{D}$. If there exists a point $z_{1}, 0<\left|z_{1}\right|<1$ and the sector $S_{\delta}\left(z_{1}\right)$, for which

$$
\max \left\{z \in S_{\delta}\left(z_{1}\right):|f(z)|\right\}=\left|f\left(z_{1}\right)\right|
$$

where $z_{1}=\left|z_{1}\right| e^{i \theta_{1}}$ and

$$
S_{\delta}\left(z_{1}\right)=\left\{r e^{i \theta}: 0 \leq r \leq\left|z_{1}\right|,\left|\theta-\theta_{1}\right|<\delta\right\}
$$

then we have

$$
\frac{z_{1} f^{\prime}\left(z_{1}\right)}{f\left(z_{1}\right)} \in \mathbb{R}, \quad \frac{z_{1} f^{\prime}\left(z_{1}\right)}{f\left(z_{1}\right)} \leq p
$$

moreover

$$
\mathfrak{R e}\left\{1+\frac{z_{1} f^{\prime \prime}\left(z_{1}\right)}{f^{\prime}\left(z_{1}\right)}\right\} \leq \mathfrak{R e}\left\{\frac{z_{1} f^{\prime}\left(z_{1}\right)}{f^{\prime}\left(z_{1}\right)}\right\}=\frac{z_{1} f^{\prime}\left(z_{1}\right)}{f^{\prime}\left(z_{1}\right)} \leq p .
$$

For some related results we refer to $[7,8,9]$. 


\section{References}

[1] I. S. Jack, Functions starlike and convex of order $\alpha$, J. London Math. Soc. 3(1971) 469-474.

[2] S. S. Miller, P. T. Mocanu, Differential subordinations and univalent functions, Michigan Math. J. 28(1981) 151-171.

[3] S. S. Miller, P. T. Mocanu, On some classes of first order differential subordinations and univalent functions, Michigan Math. J. 32(1985) 185195.

[4] S. S. Miller, P. T. Mocanu, Differential Subordinations: Theory and Applications, Series of Monographs and Textbooks in Pure and Applied Mathematics, Vol. 225, Marcel Dekker Inc., New York / Basel 2000.

[5] M. Nunokawa, On Properties of Non-Carathéodory Functions, Proc. Japan Acad. Ser. A 68(6)(1992) 152-153.

[6] M. Nunokawa, On the order of strongly starlikeness of strongly convex functions, Proc. Japan Acad. Ser. A 69(7)(1993) 234-237.

[7] M. Nunokawa, J. Sokół, On some geometric properties of multivalent functions, Journal of Inequalities and Applications, 2015, 2015:300.

[8] M. Nunokawa, J. Sokół, On some differential subordinations, Studia Scient. Math. Hungarica 54(4)(2017) 1-10.

[9] M. Nunokawa, J. Sokół, N. E. Cho, Some applications of Nunokawa's lemma, Bull. Malaysian Math. Sci. Soc. 40(4)(2017) 1791-1800.

Mamoru NUNOKAWA,

University of Gunma,

Chuou-Ward, Chiba, 260-0808, Japan.

Email: mamoru_nuno@doctor.nifty.jp

Janusz SOKÓ£,

Faculty of Mathematics and Natural Sciences,

University of Rzeszów,

ul. Prof. Pigonia 1, 35-310 Rzeszów, Poland.

Email: jsokol@ur.edu.pl 